\title{
Cardiac Function and Exercise Capacity of Diabetic Patients
}

\author{
Sadahiko Uснімото, Kei Tsumura, Naotoshi Yamashita, Hiroshi Kishimoto, \\ Shoji FujIOKA, Kyoko IzUmotani, and Hirotoshi MORII \\ Second Department of Internal Medicine, Osaka City University Medical School, \\ 1-5-7, Asahi-machi, Abeno-ku, Osaka 545, Japan
}

\begin{abstract}
Summary To evaluate the relationship between cardiac function at rest and the exercise capacity of diabetic patients, left ventricular function and exercise capacity were evaluated in 15 non-insulin-dependent male diabetic patients. Isovolumic relaxation time (IRT) and the PEP/ LVET ratio were obtained by simultaneous echophonocardiograms, electrocardiograms, and recordings of the carotid arterial pulse. $\dot{\mathrm{VO}}_{2}$ at anaerobic threshold was obtained from a cycle ergometer exercise test with expired gas analysis. Patients were divided into two groups: those with IRT $<90 \mathrm{msec}$ (Group 1) and those with IRT $\geqq 90 \mathrm{msec}$ (Group 2). Group 2 patients had a lower $\dot{\mathrm{VO}}_{2}$ at anaerobic threshold than Group 1 (Group 1: 17. $4 \pm 3.6$, Group 2: $12.9 \pm 2.5 \mathrm{ml} / \mathrm{min} / \mathrm{kg} ; \mathrm{M} \pm \mathrm{SD}, p<0.05$ ). There was a good correlation between the IRT at rest and $\Delta \mathrm{HR}$, defined as the change in heart rate from rest to anaerobic threshold $(r=0.666, p<0.01)$, and between $\Delta \mathrm{HR}$ and the beat-to-beat variation in $\mathrm{R}-\mathrm{R}$ interval at rest $(r=0.637, p<0.02)$. There was also a good correlation between $\dot{\mathrm{VO}}_{2}$ at anaerobic threshold and IRT $(r=0.555, p<0.05)$, and between $\dot{\mathrm{VO}}_{2}$ at anaerobic threshold and $\Delta \mathrm{HR}(r=$ $0.858, p<0.01)$. Our data suggests that both left ventricular diastolic function and cardiac sympathetic nervous system reflexes, reflected by $\Delta \mathrm{HR}$, may affect the exercise capacity of diabetic patients. Thus, IRT obtained by a non-invasive method may provide a useful index for cardiovascular response to exercise in diabetic patients without coronary artery disease.
\end{abstract}

Key Words exercise capacity, anaerobic threshold, cardiac function, autonomic nervous system

Patients with diabetes mellitus generally have reduced exercise capacity $(1,2)$. The possible existence of cardiac dysfunction and impaired autonomic nervous reflexes has been suggested to contribute to this reduced capacity. Previous studies have shown that increases in stroke volume (3) and heart rate (4) during exercise were diminished in diabetic patients with cardiac autonomic neuropathy, resulting in a reduction of increases in cardiac output during exercise $(3,5)$. Diabetic patients frequently have left ventricular dysfunction due to diabetic cardiomyopathy, even at an early stage of the disease $(6-10)$. Impairment of the autonomic nervous system is a complication of diabetes. Although cardiac function is one factor that affects exercise capacity, the relationship between this 
factor and exercise capacity is still unclear in diabetic patients.

The objective of this study was to elucidate the relationship between cardiac function at rest and the exercise capacity of diabetic patients.

\section{PATIENTS AND METHODS}

Patients. A total of 15 non-insulin-dependent male diabetic patients who ranged in age from 26 to 70 years were admitted to the study. The duration of diabetes mellitus ranged from 1 to 22 years. Patients were divided into two groups according to their isovolumic relaxation time (IRT), determined by echophonocardiography. Group 1 consisted of 9 patients with an IRT $<90 \mathrm{msec}$ who had almost normal left ventricular diastolic function, while Group 2 consisted of 6 patients with an IRT $\geqq 90 \mathrm{msec}$ who had an obvious abnormality of left ventricular diastolic function.

The age and body weight of the two groups did not differ significantly; however, the duration of diabetes in group 2 exceeded that of group 1 . There was no significant difference between the two groups with regard to fasting blood glucose and hemoglobin A1c levels. The beat-to-beat variation in $\mathrm{R}-\mathrm{R}$ interval (CVRR) at rest was longer in group 1 than in group 2 (Table 1).

None of the patients studied had signs or symptoms of coronary artery disease, or of other cardiovascular diseases, or had a history of taking digitalis or beta-blockers. However, one patient had received diuretics for congestive heart failure.

\section{METHODS}

All subjects were evaluated by echocardiography and cycle ergometer exercise testing during a stable state of diabetic control 1 or 2 weeks after their admission.

Echocardiography. The isovolumic relaxation time, systolic time intervals, and left ventricular dimensions were obtained by simultaneous echocardiograms (SSH 160A, Toshiba, Tokyo, Japan), phonocardiograms, electrocardiograms, and recordings of the

Table 1. Clinical Details of 15 Evaluated Diabetics.

\begin{tabular}{lcc}
\hline & Group 1 & Group 2 \\
\hline No. of patients & 9 & 6 \\
Mean age (yr) & $47 \pm 13$ & $58 \pm 5$ \\
Body weight (kg) & $62.8 \pm 16.6$ & $60.5 \pm 11.9$ \\
Duration of diabetes (yr) & $4.9 \pm 5.9^{*}$ & $14.0 \pm 6.8$ \\
FBS (mg/dl) & $149 \pm 62$ & $124 \pm 45$ \\
HBA1c (\%) & $8.8 \pm 2.3$ & $8.7 \pm 2.1$ \\
CVRR (\%) & $3.0 \pm 1.3^{*}$ & $1.7 \pm 0.8$ \\
\hline
\end{tabular}

Means \pm SD. ${ }^{*} p<0.05$ Group 1 vs. Group 2; FBS, fasting blood sugar level;

HBA1c, hemoglobin A1c;CVRR, beat-to-beat variation in R-R interval at rest. 
carotid arterial pulse. The isovolumic relaxation time (IRT), left ventricular ejection time (LVET), pre-ejection time (PEP), end-diastolic left ventricular dimensions(LVDd) and end-systolic left ventricular dimensions (LVDs) were measured by previously reported methods $(6,7,11)$. The following three parameters were derived: IRT, PEP/LVET ratio, and fractional shortening $(\% \mathrm{FS})=(\mathrm{LVDd}-\mathrm{LVDs}) / \mathrm{LVDd} * 100$.

Exercise test. A cycle ergometer (Aerobic Exercise Ergometer, Combi, Tokyo, Japan) was used for the exercise studies. The exercise protocol consisted of a symptomlimited ramp exercise test. After a rest for 2 minutes on the ergometer, exercise began with a 2-minute warm-up at $20 \mathrm{~W}$ and $60 \mathrm{rpm}$, followed by $1-\mathrm{W}$ incremental loading every 3 seconds. The heart rate was monitored and $\dot{\mathrm{V}} \mathrm{O}_{2}, \dot{\mathrm{V}} \mathrm{CO}_{2}$, and $\dot{\mathrm{V}} \mathrm{E}$ were measured by the breath-by-breath method, at rest and throughout the exercise period, using a metabolic measurement cart (MMC 4400 tc, Sensor Medics, California, U.S.A.). The anaerobic threshold point(AT) was determined visually and expressed at $\dot{\mathrm{V}} \mathrm{O}_{2}(\mathrm{ml} / \mathrm{min} / \mathrm{kg})$, using the following criteria: the $\dot{\mathrm{V}} / \dot{\mathrm{V}} \mathrm{O}_{2}$ curve, which was flat or decreasing, began to rise as the $\dot{\mathrm{VE}} / \dot{\mathrm{V} C O}{ }_{2}$ curve remained constant or decreased (12). AT-HR was defined as the heart rate at AT and $\triangle \mathrm{HR}$ was defined as the change in heart rate from rest to AT.

All data are shown as mean \pm SD. Statistical analysis was performed according to Student's $t$-test. A $p$ value of $<0.05$ was considered to be statistically significant.

\section{RESULTS}

Fractional shortening was significantly lower in Group 2 than in Group $1(p<0.05)$
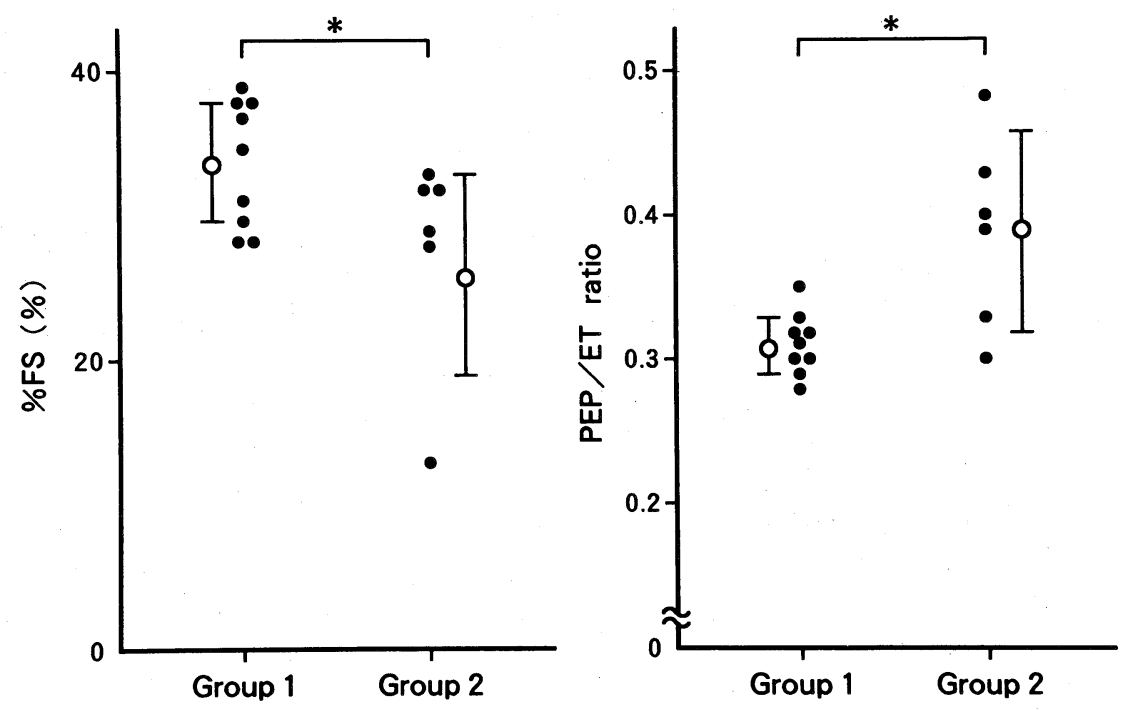

Fig. 1. Fractional shortening and PEP/LVET ratio in 15 diabetic patients. Data are expressed as mean $\pm \mathrm{SD} .{ }^{*} p<0.05$. 


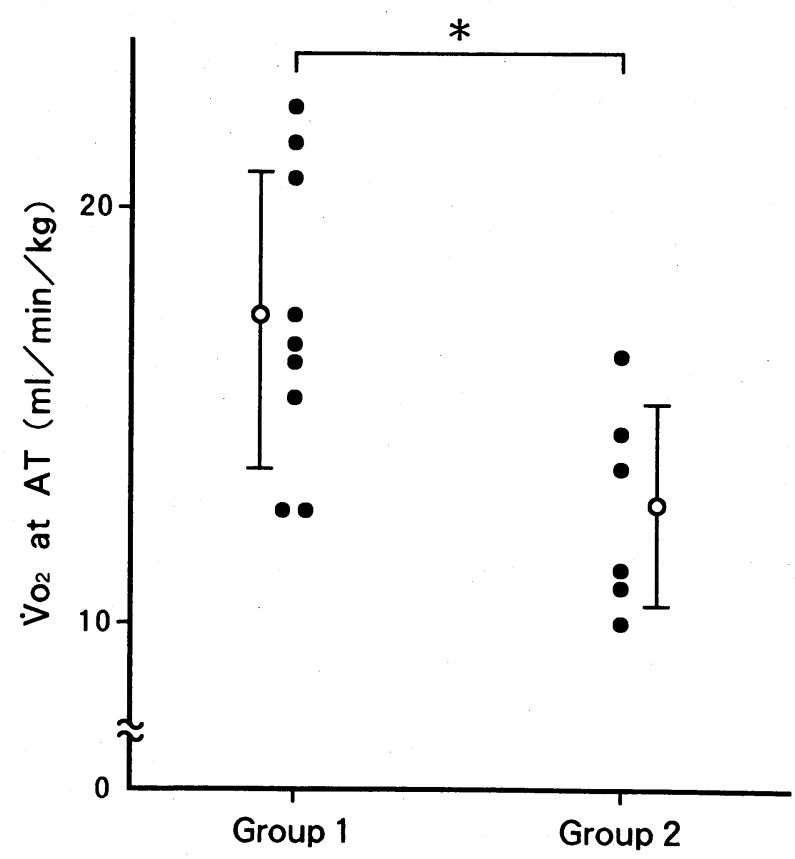

Fig. 2. $\dot{\mathrm{V}} \mathrm{O}_{2}$ at anaerobic threshold in 15 diabetic patients. Data are expressed as mean $\pm \mathrm{SD}$. ${ }^{*} p<0.05$.

and the PEP/LVET ratio was significantly higher in Group 2 than Group $1(p<0.05)$. The $\mathrm{PEP} / \mathrm{LVET}$ ratio of all patients in Group 1 was less than 0.35 , while that of 4 patients in Group 2 was greater than 0.35 , indicating the presence of left ventricular systolic dysfunction (Fig.1).

Figure 2 shows that $\dot{\mathrm{VO}} 2$ at AT was significantly lower in Group 2 than in Group 1 ( $p$ $<0.05)$.

Figure 3 shows that AT-HR and $\triangle \mathrm{HR}$ were significantly lower in Group 2 than in Group $1(p<0.01)$. Furthermore, there was a good correlation between IRT and AT-HR $(r=-0.812)$ and between IRT and $\Delta$ HR $(r=-0.666)$ (Fig.4).

Figure 5 shows the relationship between CVRR and AT-HR and between CVRR and $\Delta \mathrm{HR}$. There was a good correlation between CVRR and $\Delta \mathrm{HR}(r=0.637)$, but not between CVRR and AT-HR.

Figure 6 shows the relationship between IRT and $\dot{\mathrm{V}} \mathrm{O}_{2}$ at AT and between $\Delta \mathrm{HR}$ and $\dot{\mathrm{V}} \mathrm{O}_{2}$ at AT. There was a good correlation between both IRT and $\dot{\mathrm{V}} \mathrm{O}_{2}$ at AT $(r=-0.555)$ and between $\Delta \mathrm{HR}$ and $\dot{\mathrm{VO}}_{2}$ at $\mathrm{AT}(r=0.858)$.

\section{DISCUSSION}

The exercise capacity of diabetic patients has been reported to be reduced, especially 

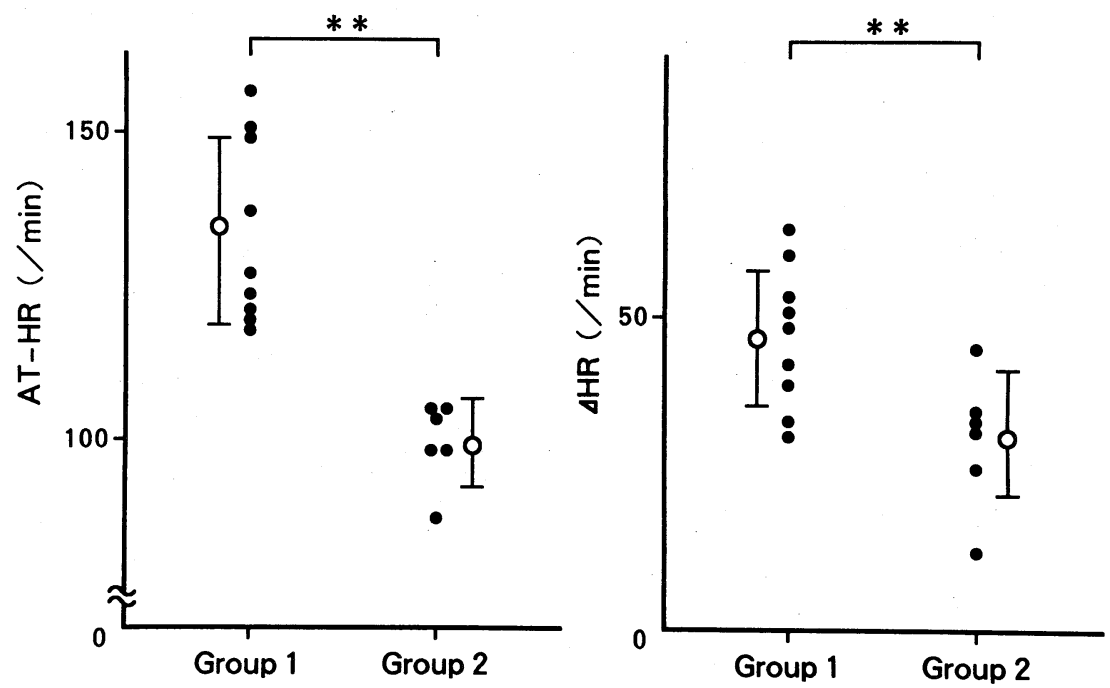

Fig. 3. AT-HR and $\Delta \mathrm{HR}$ in 15 diabetic patients. Data are expressed as mean $\pm \mathrm{SD} .{ }^{* *} p<0.01$.

in those with cardiac autonomic neuropathy. Hilsted et al. (1) reported that the maximal exercise capacity of such patients who had decreased the beat-to-beat variation in R-R interval was reduced. Kremser et al. (2) reported that the maximal $\dot{\mathrm{V}} \mathrm{O}_{2}$ was reduced in diabetic patients with cardiac autonomic neuropathy as compared to normal subjects and diabetic patients without sensory or autonomic neuropathy, and that $\dot{\mathrm{V}} \mathrm{O}_{2}$ at anaerobic threshold was similar for the three groups.

We were unable to measure maximal exercise capacity in our patients as their exercise was ended before they had achieved maximal exercise resulting in dyspnea or leg fatigue. Our study showed that diabetic patients with left ventricular diastolic dysfunction had a shorter beat-to-beat variation in R-R interval and a lower $\dot{\mathrm{VO}}_{2}$ at AT than did the diabetic patients with normal left ventricular diastolic function. Patients with a prolonged IRT did not always have a short CVRR, so that diabetic patients with both a prolonged IRT and a short CVRR had a low $\dot{\mathrm{VO}}_{2}$ at $\mathrm{AT}$, indicating reduced exercise capacity.

To explain the causes of reduced exercise capacity in diabetic patients with cardiac autonomic neuropathy, it has been suggested that the increase in stroke volume, and thus in cardiac output, was reduced during exercise (3), suggesting that there was a reduced left ventricular performance. There is considerable evidence that diabetic patients have abnormalities of left ventricular function in the absence of clinical heart disease $(16-18)$. Rubler et al. (16) reported four patients with diabetic glomerulosclerosis who presented with cardiomegaly and congestive heart failure of unknown cause. Ahmed (6) and Shapiro (7-10), using a non-invasive method, demonstrated an impairment of left ventricular function in diabetic patients with no coronary artery disease. Delia et al. (19) reported diabetic patients with an abnormal pressure-volume curve of the left ventricle but with no coronary artery disease. 

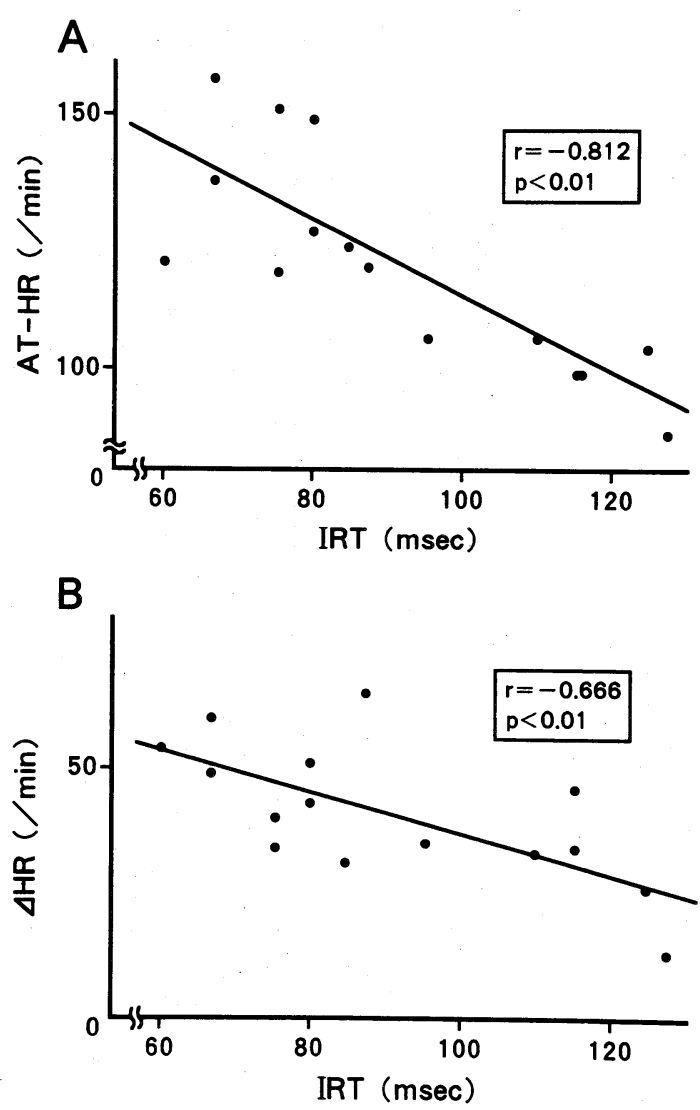

Fig. 4. A: Regression analysis of AT-HR on IRT in 15 diabetic patients. B: Regression analysis of $\triangle \mathrm{HR}$ on IRT in 15 diabetic patients.

Kahn et al. (4) reported that the increase in heart rate during exercise was decreased in diabetic patients with cardiac autonomic neuropathy. Autonomic nervous system reflexes are important in the cardiovascular response to exercise, and abnormalities in autonomic nervous system function would be expected to produce a deranged regulation of responses to exercise (5).

While there are many reports on cardiovascular performance during exercise in diabetics, there are none of the relationship between left ventricular function at rest and exercise capacity. Although exercise therapy is very important for diabetic patients, the examination of cardiac function and exercise capacity is necessary, since some diabetic patients with no coronary artery disease are at risk of sudden cardiac death during exercise. If we could evaluate the cardiac function and exercise capacity of such patients by a non-invasive method, it might easier to determine whether or not exercise is contraindicated for them.

In this study we attempted to demonstrate a relationship between left ventricular 

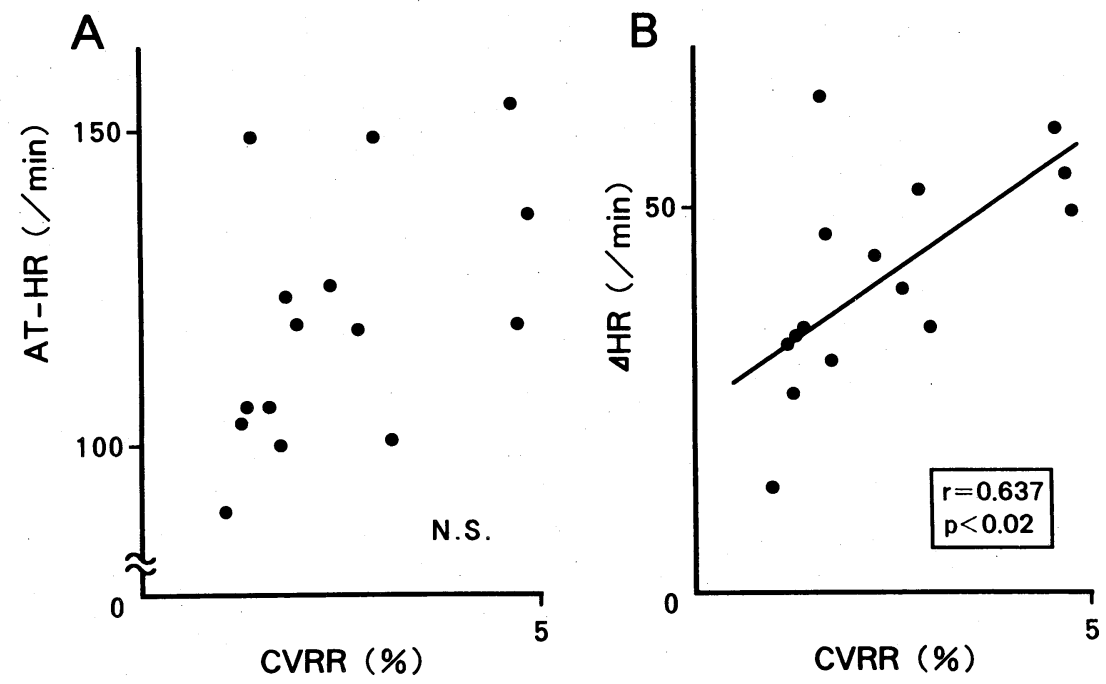

Fig. 5. A: Regression analysis of AT-HR on CVRR in 15 diabetic patients. B: Regression analysis of $\triangle \mathrm{HR}$ on CVRR in 15 diabetic patients.

function at rest and exercise capacity. Diabetic patients with obvious left ventricular diastolic dysfunction had a reduced exercise capacity. The increase in heart rate during exercise is determined by many factors, particularly by the function of the sympathetic nervous system. In our study there was a good correlation between $\triangle \mathrm{HR}$ and CVRR, one of the indices of parasympathetic nervous system function, suggesting that $\Delta H R$ may be an index of sympathetic nervous system function or of myocardial cell response to catecholamine. There was no significant correlation between AT-HR and CVRR, as AT$\mathrm{HR}$ is determined by both the parasympathetic and sympathetic nervous systems. That is, a clinically diminished heart rate during exercise seems to be a result of the impairment of sympathetic nervous system reflexes. Thus, diabetic patients with left ventricular diastolic dysfunction have both a reduction in their exercise capacity and an impairment of their sympathetic nervous system reflexes.

There are a few reports indicating that the abnormal left ventricular function of diabetic patients is related not only to the severity of their microangiopathy $(9,16,18)$, but also to their state of diabetic control (13-15). The prolonged IRT in our patients would appear to indicate that left ventricular function was impaired due mainly to microangiopathy, since there was no difference in the state of diabetic control between the two groups.

There was a good correlation between left ventricular diastolic function and exercise capacity, and a good correlation between sympathetic nervous system reflexes and exercise capacity. Our results suggest that diabetic patients with impaired left ventricular diastolic function have impaired sympathetic nervous system reflex responses to exercise, and thus both factors affect their exercise capacity. The isovolumic relaxation time is 

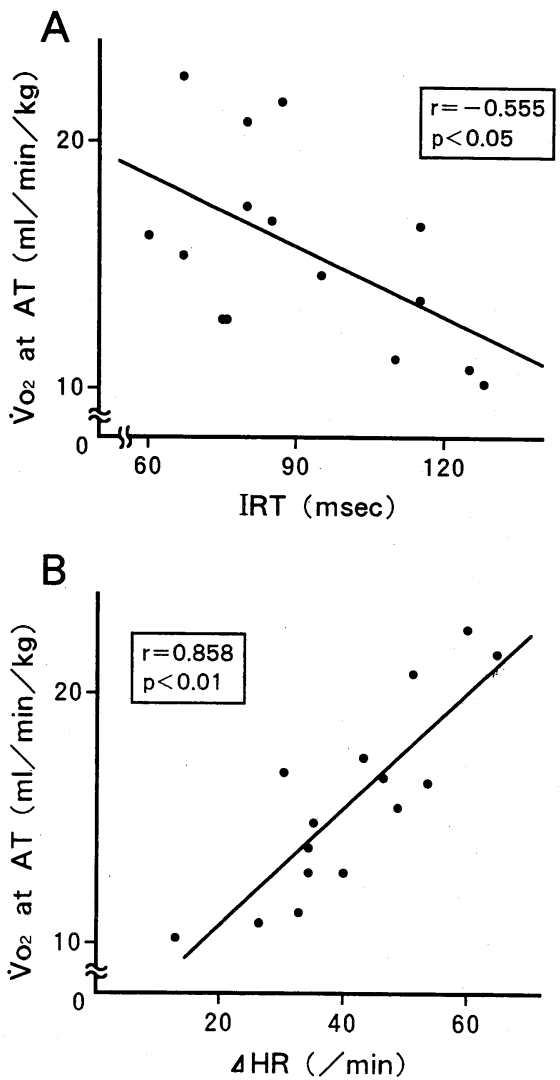

Fig. 6. A: Regression analysis of $\mathrm{V}_{2}$ at AT on IRT in 15 diabetic patients. B: Regression analysis of $\dot{\mathrm{VO}}_{2}$ at AT on $\Delta \mathrm{HR}$ in 15 diabetic patients.

prolonged earlier than the PEP/LVET ratio in diabetic patients (5) and is thus a more sensitive index of impaired left ventricular function than this ratio. Furthermore, the isovolumic relaxation time is related to exercise capacity and sympathetic nervous system reflex responses to exercise in diabetic patients measured by a non-invasive method; this measurement appears to provide a useful index for cardiovascular response to exercise in diabetic patients with no coronary artery disease.

\section{REFERENCES}

1) Hilsted, J., Galbo, H., and Christensen, N.J. (1979): Impaired cardiovascular responses to graded exercise in diabetic autonomic neuropathy. Diabetes, 28, 313-319.

2) Kremser, C.B., Levitt, N.S., Borow K.M., et al. (1988): Oxygen uptake kinetics during exercise 
in diabetic neuropathy. J. Appl. Physiol. , 65, 2665-2661.

3) Hilsted, J., Galbo, H., Christensen N.I. (1982): Haemodynamic change during graded exercise in patients with diabetic autonomic neuropathy. Diabetologia, 22, 318-323.

4) Kahn, J.K., Zola, B., Juni J.E. et al. (1986): Decreased exercise heart rate and blood pressure response in diabetic subjects with cardiac autonomic neuropathy. Diabetes Care, 9, 389-394.

5) Roy, T.M., Peterson, H.R., Snider, H.L.(1989): Autonomic influence on cardiovascular performance in diabetic subjects. Am.J. Med., 87, 382-388.

6) Ahmed, S.S., Jaferi, G.A., Narang, R.M. et al. (1975): Preclinical abnormalities of left ventricular function in diabetes mellitus. Am. Heart J., 89, 153-158.

7) Shapiro, L.M., Leatherdale, B.A., Coyne, M.E., et al. (1980): Prospective study of heart disease in untreated maturity onset diabetics. Br. Heart J., 44, 342-348.

8) Shapiro, L.M., Howat, A.P., and Calter, M.N. (1981): Left ventricular function in diabetes mellitus 1: Methodology, and prevalence and spectrum of abnormalities. Br. Heart J., 45, $122-128$.

9) Shapiro, L.M., Leatherdale, B.A., Mackinnon, J., et al. (1981): Left ventricular function in diabetes mellitus 2: Relation between clinical features and left ventricular function. Br. Heart J., 45, 129-132.

10) Shapiro, L.M. (1982): Echocardiographic features of impaired ventricular function in diabetes mellitus. Br. Heart J., 46, 439-444.

11)Weissler, A.M., Harris, W.S., and Schoenfeld, C.D. (1968): Systolic time intervals in heart failure in man. Circulation, 37, 149-159.

12) Wasserman, K., Mcllroy, M.B., Alto, P., et al. (1964): Detecting the threshold of anaerobic metabolism in cardiac patients during exercise. Am. J. Cardiol., 14, 844-852.

13) Uusitupa, M., Siitonen, O., Aro, A., et al. (1983): Effect of correction of hyperglycemia on left ventricular function in non-insulin-dependent (Type 2) diabetics. Acta Med. Scand., 213, 363-368.

14) Sykes, C.A., Wright, A.D., Malins, J.M., et al. (1977): Changes in systolic time intervals during treatment of diabetes mellitus. Br. Heart J., 38, 255-259.

15) Sasakawa, O., Fujii, S., Nogi, O., et al. (1984): Left ventricular function in patients with diabetes mellitus. Jpn. J. Med., 23, 34-39.

16) Rubuler, S., Dlugash, J., and Yuceoglu, Y.Z. et al. (1972): New type of cardiomyopathy associated with diabetic glomerulosclerosis. Am.J. Cardiol., 30, 595-602.

17) Kannel, W.B., Hjortland, M., and Castelli, W.P. (1974): Role of diabetes in congestive heart failure: The Framingham study. Am. J. Cardiol., 34, 29-34.

18) Hamby, R.I., Zoneraich, S., and Sherman, L. (1974): Diabetic cardiomyopathy. J. Am. Med. Assoc., 229, 1749-1754.

19) Delia, J.A., Weinrauch, L.A., Healy, R.W., et al. (1979):Myocardial dysfunction without coronary artery disease in diabetic renal failure. Am. J. Cardiol., 43, 193-199. 